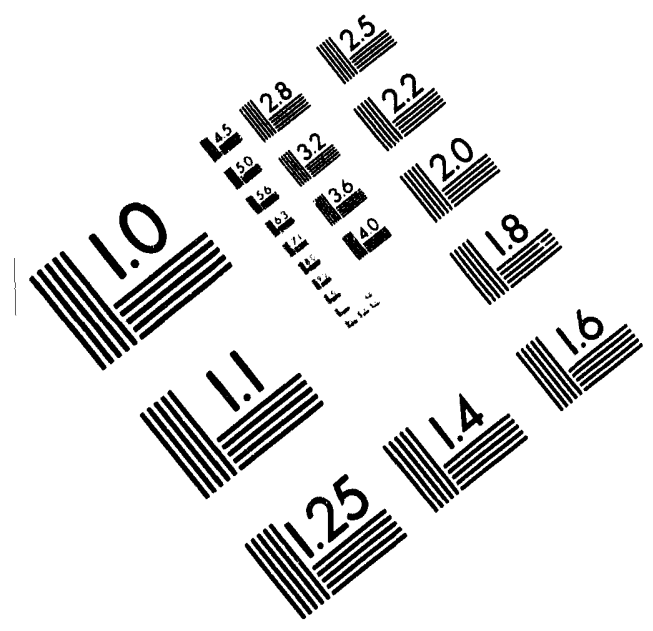

-JNI • $\exists$ OUWI 0

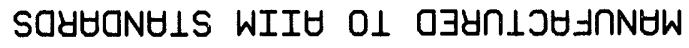
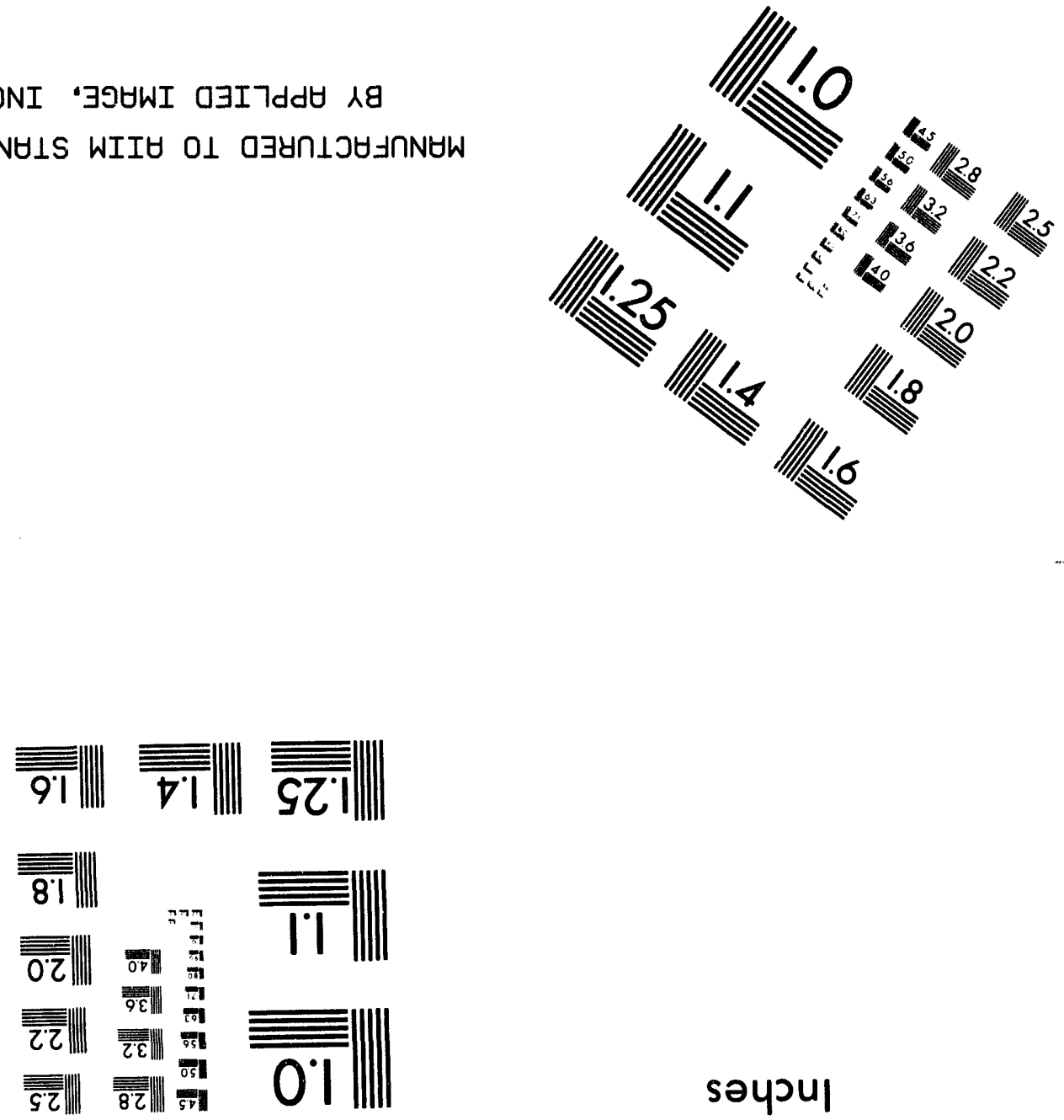

sәyวul
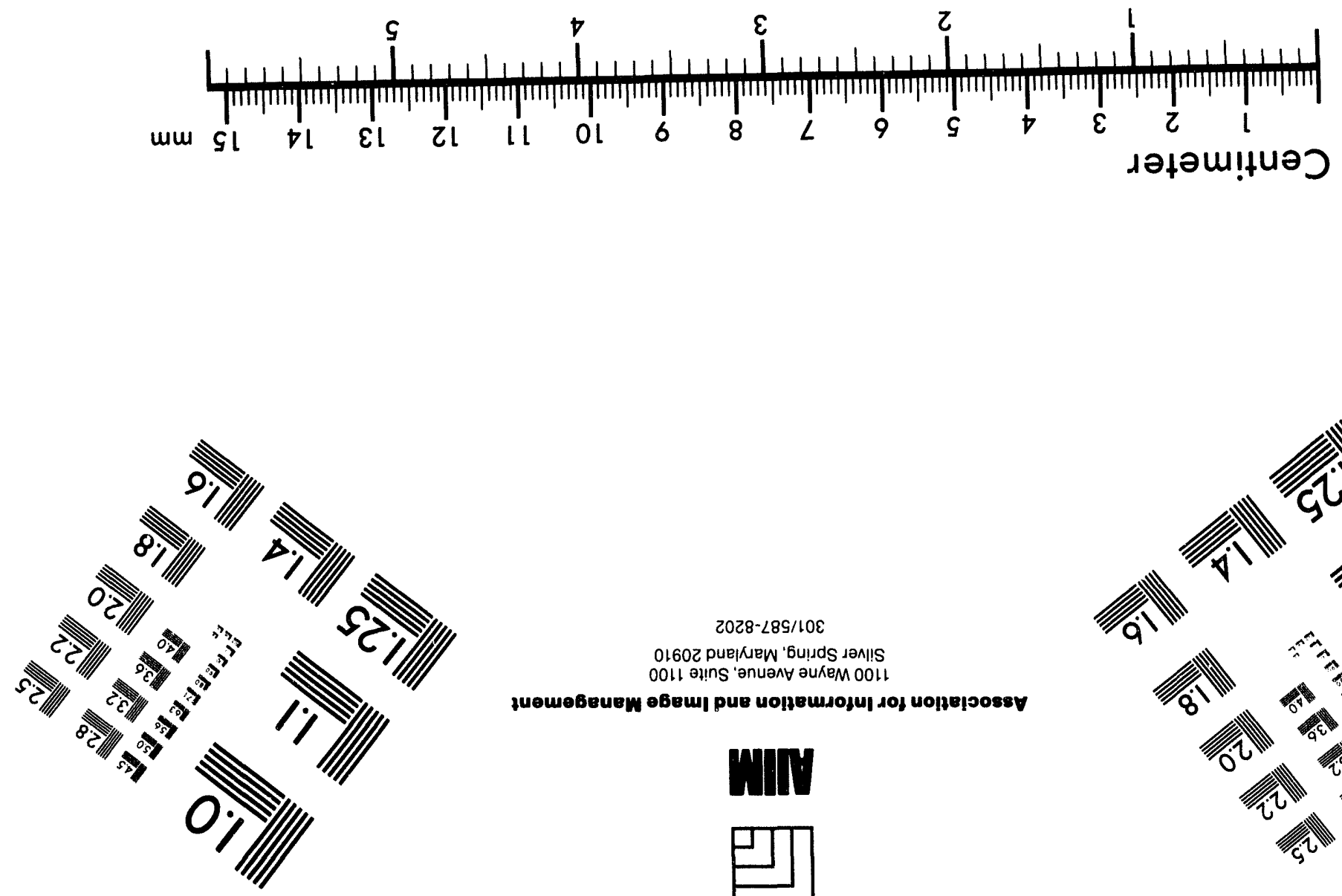

20Z8- $\angle 8 \mathrm{~S} / \mathrm{LOE}$

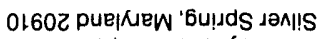

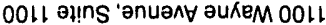

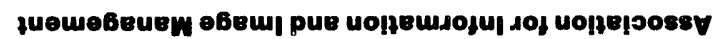

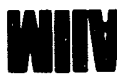

$\square$

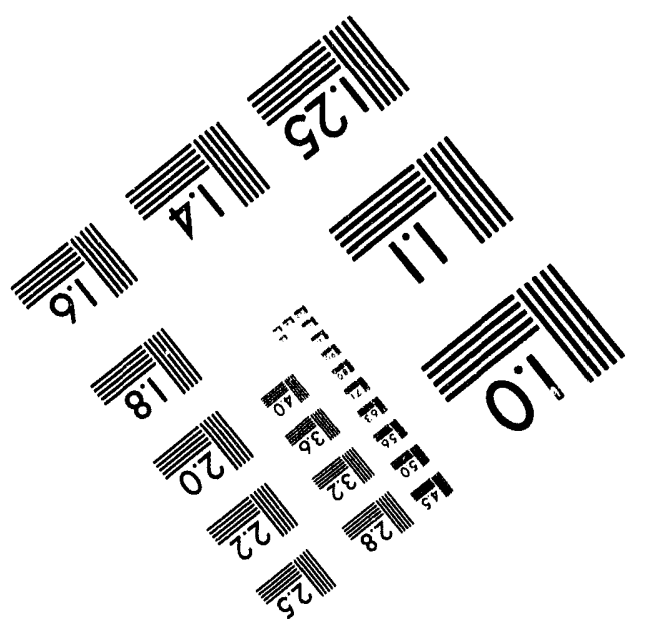



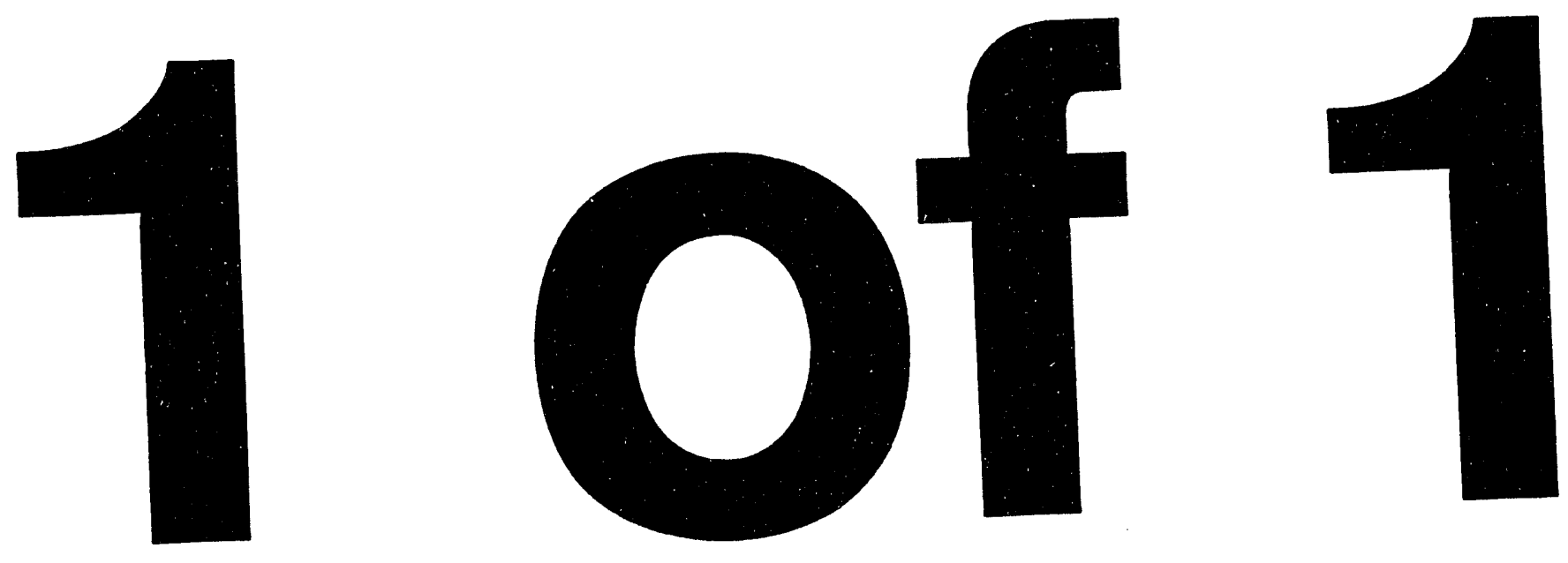


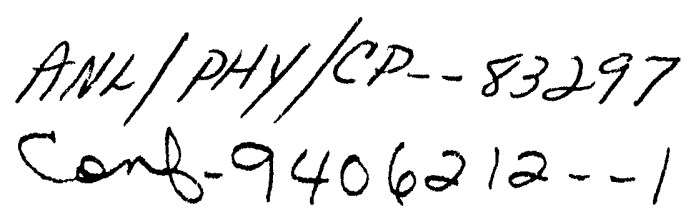

SEARCH FOR OCTUPOLE DEFORMATION IN NEUTRON RICH Xe ISOTOPES

\author{
M. Bentaleb, N. Schulz, E. Lubkiewicz *
}

Centre de Recherches Nucléaires, IN2P3-CNRS/Université Louis Pasteur, B.P.20, 67037

Strasbourg, France

J.L. Durell, F. Lidén, C.J. Pearson, W.R. Phillips, J. Shannon, B.J. Varley

Department of Physics, University of Manchester, M13 9PL, United Kingdom

C.J. Lister *

A.W. Wright Nuclear Structure Laboratory, Yale University, New Haven, CT 06511, USA

I. Ahmad, L.R. Morss, K.L. Nash, C.W. Williams

Argonne National Laboratory, Argonne, IL 60439, USA

Abstract : A search for octupole deformation in neutron rich Xe isotopes has been conducted through gamma-ray spectroscopy of primary fragments produced in the spontaneous fission of ${ }^{248} \mathrm{Cm}$. The spectrometer consisted of the Eurogam array and a set of 5 LEPS detectors. Level schemes were constructed for Xe isotopes with masses ranging from 138 to 144. Except for ${ }^{139} \mathrm{Xe}$, none of them exhibit an alternating parity quasimolecular band, $a$ * feature usually encountered in octupole deformed nuclei.

Substantial evidence for reflection asymmetric shape in the intrinsic system of the nucleus exists for the light actinide nuclei. Experimentally it manifests itself by the presence of alternating parity quasimolecular bands, of strong El transitions and of parity doublets. Recently a new island of nuclei which show characteristics of octupole deformation has been discovered in the vicinity of neutron-rich $\mathrm{Ba}$ nuclei ${ }^{1,2}$ ). Reflection asymmetry results there from the octupole coupling between the $i_{13 / 2}$ and $f_{7 / 2}$ neutron orbitals and the $h_{11 / 2}$ and $d_{5 / 2}$ proton orbitals and several theoretical studies ${ }^{3-5}$ ) have been published on octupole correlations in the neutron-rich Xe nuclei. The first approach ${ }^{3}$ ) uses the deformed shell model and includes high-multipole deformations up to $\beta_{7}$; the second one ${ }^{4}$ ) calculates shapes using the cranking model with pairing and focuses on high-spin structures. In the last approach ${ }^{5}$ ), the calculations based on the Gogny force predict the behaviour of $E 1$ transition probabilities for several nuclei. However, experimental information is available only for ${ }^{142} \mathrm{Xe}$, which in contrast to the predictions, does not exhibit properties characteristic of octupole deformation.

The decay path of the excited primary fission fragment ${ }^{142} \mathrm{Xe}$ populated in the spontaneous fission of ${ }^{248} \mathrm{Cm}$ was measured using a $\gamma$-ray facility including seven Ge-detectors and one LEPS (low energy photon spectrometer). The advent of the large $\gamma$-ray multidetector array 
Eurogam ${ }^{7}$ ) incited us to measure again the prompt $\gamma$-rays produced by a ${ }^{248} \mathrm{Cm}$ source. As far as $\mathrm{Xe}$ isotopes are concerned, new information was expected from this high statistics experiment : the observation of weak side-bands, the possibility of determining the yrast band in more exotic nuclei and above all the investigation of odd-A Xe isotopes, which are less populated than their even-even neighbours. The interest in the last point results from the prediction that the asymmetry energy is larger in the odd-A nuclei ${ }^{3}$ ).

Experiment and data analysis

The source was prepared by mixing $\sim 5 \mathrm{mg}$ of ${ }^{248} \mathrm{Cm}\left(\sim 6.3 \cdot 10^{4}\right.$ fissions/s) in the form of oxide with $65 \mathrm{mg}$ of $\mathrm{KCl}$ and compressing the mixture into a 7 -mm pellet. In this way the fission fragments are stopped in a short time $(\sim 1 \mathrm{ps})$ and almost all prompt $\gamma$-rays are emitted at rest. The ${ }^{248} \mathrm{Cm}$ source was placed in the center of the Eurogam spectrometer which in phase 1 was located at the Daresbury Nuclear Structure Laboratory. The Eurogam array, which consisted in this experiment of 45 Compton-suppressed large volume Germanium detectors, was augmented by the addition of 5 LEPS detectors. The acquisition system was triggered only when the number of Ge triggers (unsuppressed) was greater than 3 . This considerably reduced events associated with $\beta$-decay, whereas the events from prompt fission were much less affected since they have an average $\gamma$-ray multiplicity of roughly 10.

The $\gamma$-lines of interest lie on a high background and the analysis required single-, doubleand even triple-gated $\gamma$ spectra. For the last case, clean spectra were obtained but at the expense of statistics and an example of such a spectrum is shown in fig.1. Gamma-gamma angular correlations were extracted from the data for the strongest transitions and the ratio $A_{2} / A_{0}$ was determined from Legendre polynomial fits. In several cases $A_{2} / A_{0}$ values were found to be consistent either with stretched quadrupole-quadrupole or with stretched

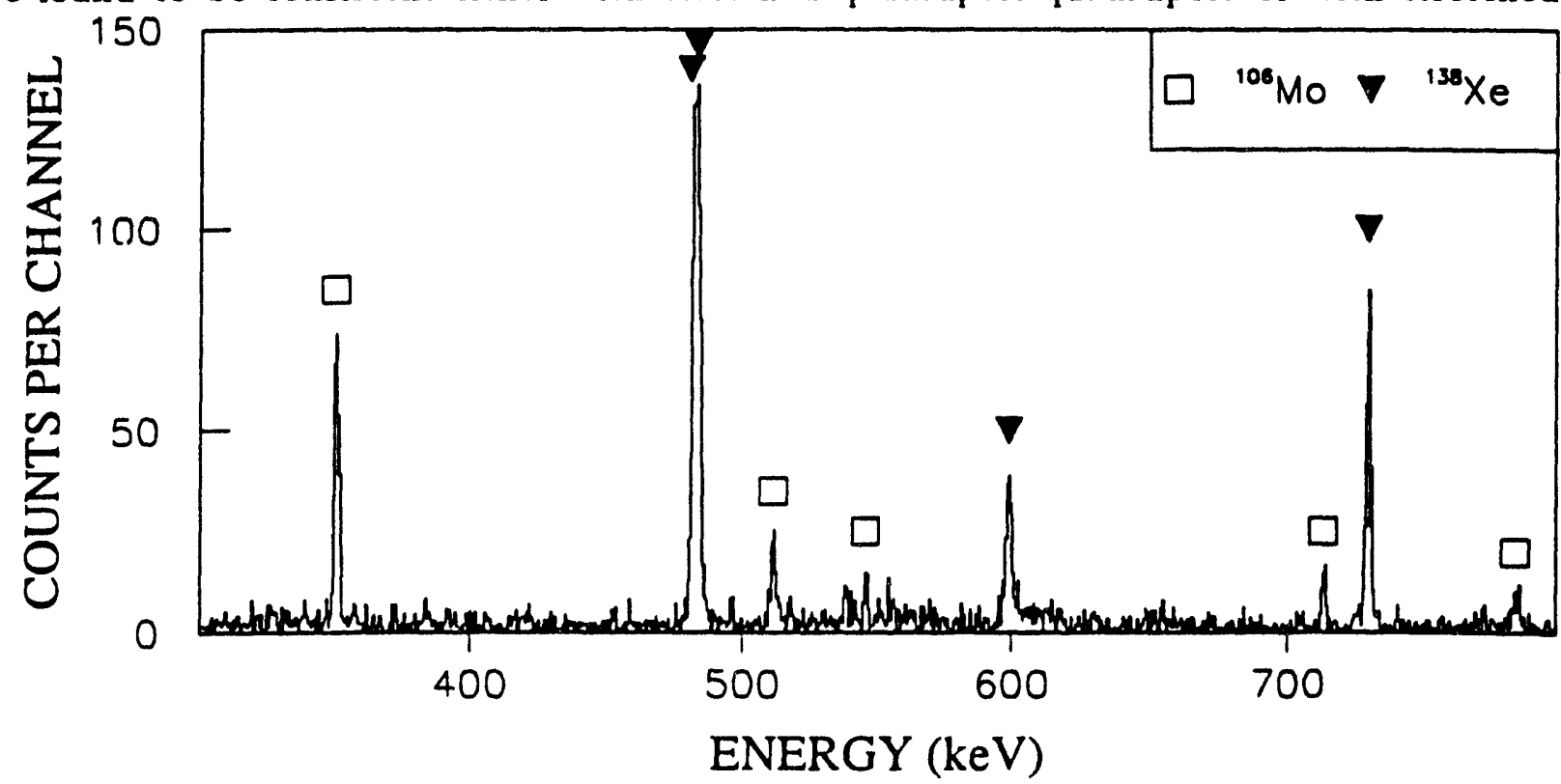

Fig.1: Quadruple-coincidence showing a Ge detector spectrum in coincidence with raw gates on the $2^{+} \rightarrow 0^{+}$transitions in both ${ }^{106} \mathrm{Mo}$ and ${ }^{138} \mathrm{Xe}$ and a background substracted gate on the $10^{+} \rightarrow 8^{+}$transition in ${ }^{138} \mathrm{Xe}$. 
dipole-quadrupole correlations, and consequently tentative spin assignments were made for several excited states knowing that fission populates preferably yrast and yrare states.

The lightest $\mathrm{Xe}$ isotopes could be identified by the presence of known $\boldsymbol{\gamma}$-lines. For heavier isotopes, an unambiguous identification technique ${ }^{6}$ ) has been used. It relies on the fact that the $\gamma$-rays in each of the Xe nuclei $(Z=54)$ are in coincidence with the $\gamma$-rays of several isotopes of the element Mo $(Z=42)$ produced simultaneously in the sporitaneous fission of ${ }^{248} \mathrm{Cm}$ $(Z=96)$, the mass of each Mo nucleus depending on the number of neutrons emitted per fission event (this number ranges typically from 1 to 5 ). The average mass of the complementary Mo fragments are plotted as a function of the known Xe isotopes and an extrapolation of the curve allows the identification of more exotic isotopes.

\section{Results and interpretation}

States with spins up to $16 \hbar$ could be observed. No transitions were known in two isotopes, ${ }^{143} \mathrm{Xe}$ and ${ }^{144} \mathrm{Xe}$, prior to this work.

Partial level schemes for the even-even Xe isotopes were deduced from the present data and three of them are shown in fig.2. For all these nuclei, the collective structure of their yrast states is attested by their positions on Mallmann's plot ${ }^{8}$ ) which represents the $\mathrm{E}\left(6_{1}^{+}\right) / \mathrm{E}\left(2_{1}^{+}\right)$. and $\mathrm{E}\left(8_{1}^{+}\right) / \mathrm{E}\left(2_{1}^{+}\right)$ratios as a function of the $\mathrm{E}\left(4_{1}^{+}\right) / \mathrm{E}\left(2_{1}^{+}\right)$values. The ${ }^{138} \mathrm{Xe}$ isotope with 84 neutrons lies close to the vibrational limit, whereas ${ }^{144} \mathrm{Xe}$ with $E\left(4_{1}^{+}\right) / E\left(2_{1}^{+}\right)=2.55$ is still far from the limit of 3.33 for a perfect rotor.

Considering the octupole degree of freedom, Martin and Robledo ${ }^{5}$ ) predicted ${ }^{140} \mathrm{Xe}$ to . belong to the pure vibrational category of nuclei. This assignment is not in contradiction with the decay scheme of ${ }^{140} \mathrm{Xe}$ where the levels shown on the left-hand side of the yrast band (fig.2) are likely candidates for the $5^{-}, 7^{-}, 9^{-}, 11^{-} \ldots$ states. Note however that according to another calculation ${ }^{4}$ ) ${ }^{140} \mathrm{Xe}$ should become octupole deformed at $\hbar \omega \simeq 0.25 \mathrm{MeV}$.

More information is now available for the side-band structure in the ${ }^{142} \mathrm{Xe}$ nucleus. Some of the yrare levels are possibly negative-parity odd-spin states, but undoubtedly no alternatingparity band appears in the level scheme. Although a minimum is predicted ${ }^{5}$ ) in the energy versus octupole moment for ${ }^{142} \mathrm{Xe}$, its amplitude is only one half the value for ${ }^{144} \mathrm{Ba}$ where some characteristics of stable octupole deformation have been observed ${ }^{1}$ ). So both experiment and a theoretical description agree to consider ${ }^{142} \mathrm{Xe}$ as an octupole soft nucleus.

${ }^{144} \mathrm{Xe}$ is the most neutron-rich $\mathrm{Xe}$ isotope for which $\gamma$-transitions could be identified. Its yield relative to ${ }^{140} \mathrm{Xe}$ reaches only $\simeq 0.02$. For this nucleus, which was predicted to be either reflection asymmetric already at the ground-state ${ }^{4}$ ) or simply octupole unstable ${ }^{5}$ ), only a single $\gamma$-cascade could be observed.

The level schemes of the two odd-A isotopes ${ }^{139} \mathrm{Xe}$ and ${ }^{143} \mathrm{Xe}$ are displayed on fig.3. From the systematics of heavier $N=35$ isotones, the $3 / 2^{-},\left(7 / 2^{-}\right),\left(11 / 2^{-}\right)$and $\left(15 / 2^{-}\right)$levels in ${ }^{139} \mathrm{Xe}$ may be identified as $\nu \mathrm{f}_{7 / 2}^{3}$ states and the lowering of the $3 / 2^{-}$state to become the ground state has been attributed to a $\nu p_{3 / 2}$ admixture in its wave function. The $(13 / 2)$ and $(17 / 2)$ spin assignments to the lowest members of the right-hand side band is an indication that these 
states, as well as higher-lying states in the same band, are plausible candidates for the $\nu f_{7 / 2}^{2} \mathrm{i}_{13 / 2}$ multiplet. More speculative is the identification of the lowest states of the left-hand band to the members of the $\nu f_{7 / 2}^{2} \mathrm{~h}_{9 / 2}$ multiplet. An alternative description to the shell-model is given by the octupole deformed (Nilsson levels ${ }^{9}$ ). The ground-state rotational band and the band immediately to the right can be understood in terms of parity doublet bands arising from the $\Omega=3 / 2$ reflection-asymmetric orbital with $\mathrm{f}_{7 / 2}$ shell-model parentage at $\beta_{2}=\beta_{3}=0$. Such a picture is supported by the strong $E 1$ transitions connecting the side-band to the ground-state band ( the average $B(E 1) / B(E 2)$ ratio is $0.3 \cdot 10^{-6} \mathrm{fm}^{-2}$ ). Note that such alternative descriptions have also been proposed in the actinides for ${ }^{217} \mathrm{Ra}$ which, like ${ }^{139} \mathrm{Xe}$, has 3 valence neutrons ${ }^{10}$ ).

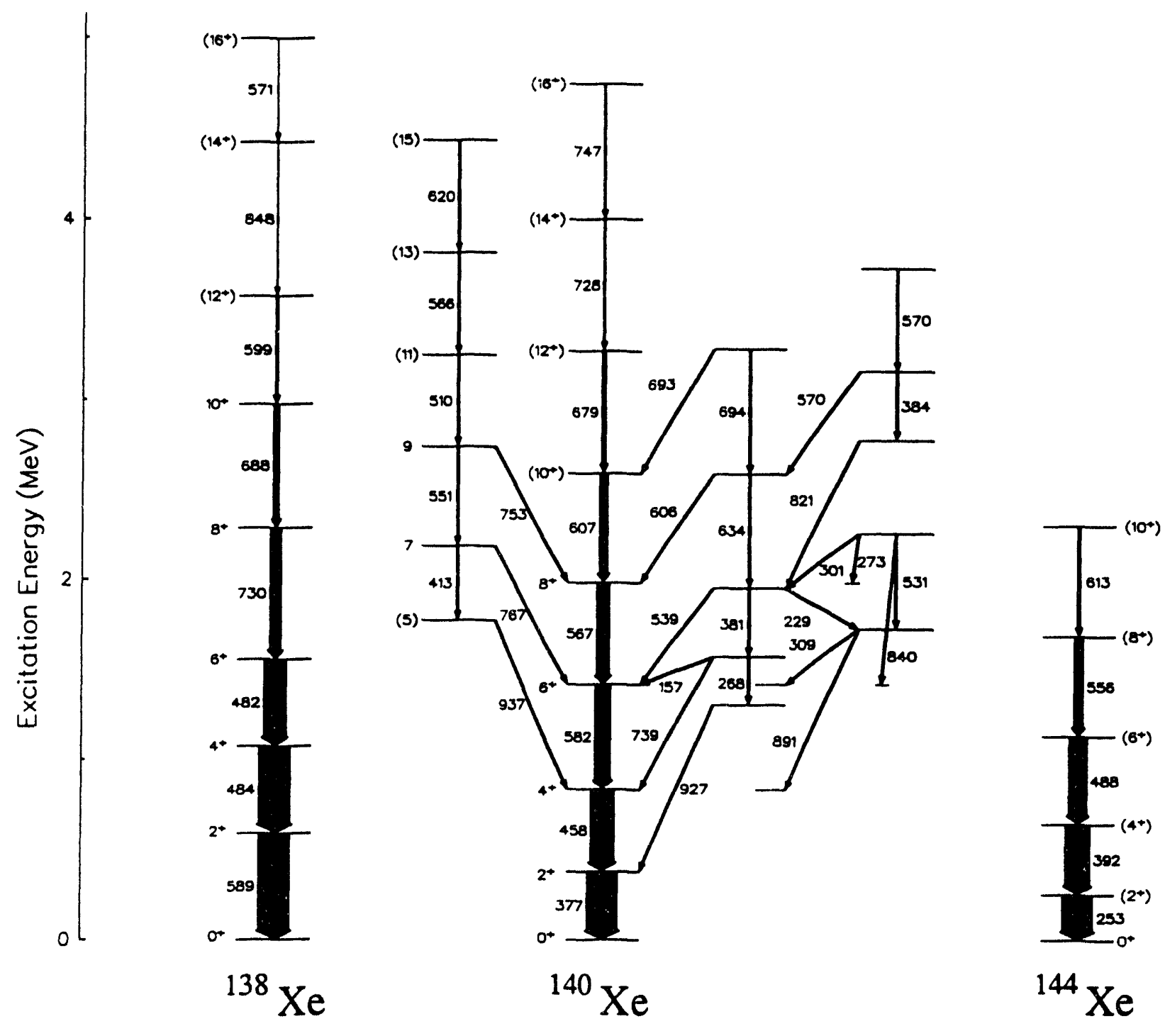

Fig.2 : Level schemes of even-even Xe nuclei studied in the present work. In each nucleus, the relative intensity of a transition is proportional to the thickness of the line representing it.

Since calculated octupole barriers in odd-A nuclei are significantly larger compared to the bariiers in even-even neighbours ${ }^{3}$ ) and since large octupole barriers imply the existence of low-lying parity doublets, it was expected to observe interlocked sets of levels in ${ }^{143} \mathrm{Ye}$. The decay pattern apparently conflicts with this expectation. The ground-state spin 
and parity values $5 / 2^{-}$cannot distinguish between the reflection-symmetric and reflectionasymmetric shapes because in both cases the ground-state may be interpreted as the $\Omega=$ $5 / 2$ orbital $^{9}$ ). However the decay of the first excited state is found to be an E1 transition from intensity balance in the gamma cascade and the presence of a low-lying first excited state with positive-parity cannot be explained in the case of $\beta_{3}=0$. In an octupole deformed nucleus however, the ground-state and first excited state could be the negative- and positive-parity members respectively of the $5 / 2^{ \pm}$doublet. The small energy difference, 79 $\mathrm{keV}$, corroborates this assumption since the energy splitting for odd-A parity doublets is proportional to the parity content of the parity-mixed orbital $\left(\langle\hat{\pi}\rangle=-0.15\right.$ for $\left.\beta_{3}=0.16\right)$.

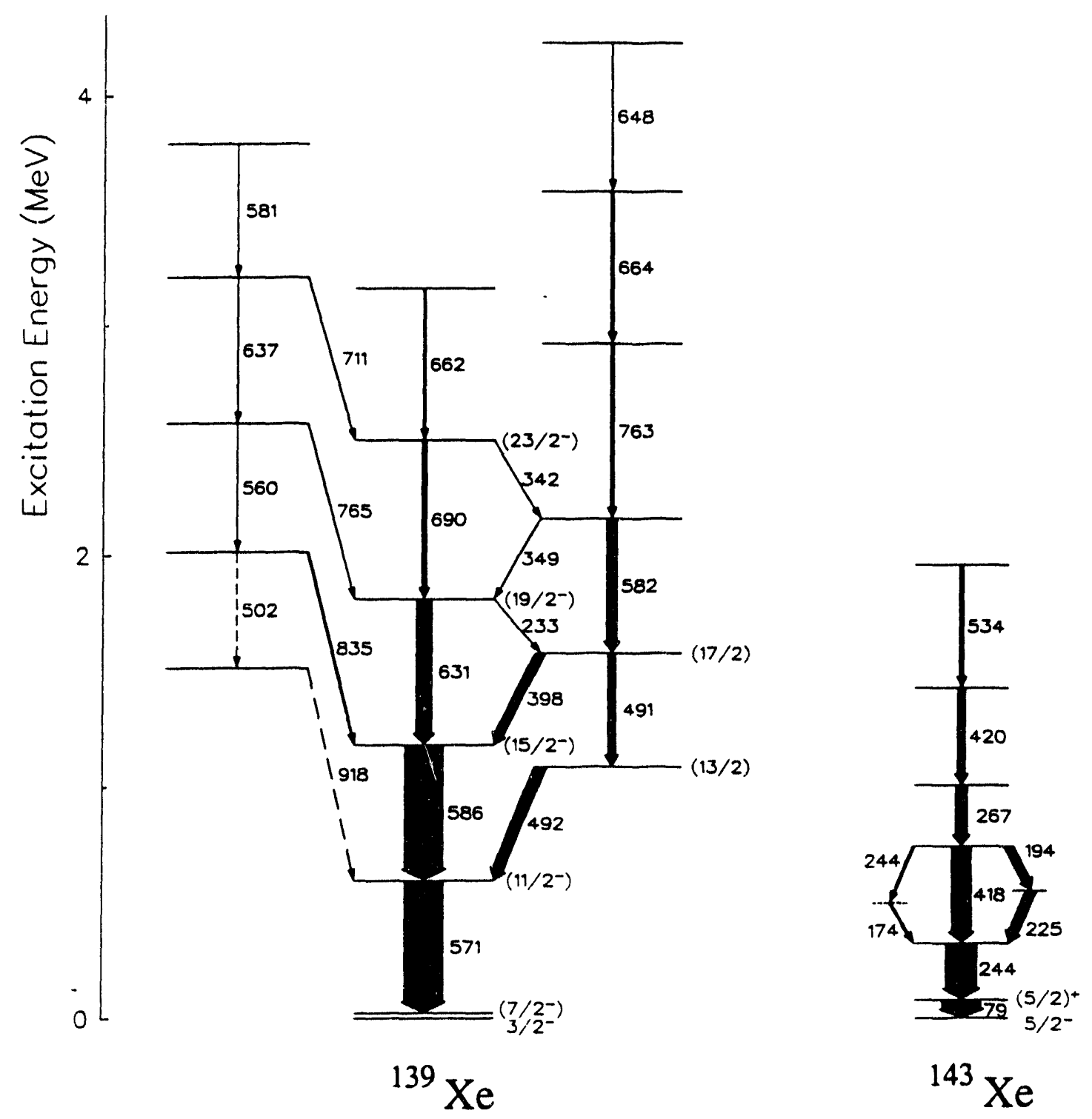

Fig.3 : Level schemes of ${ }^{139} \mathrm{Xe}$ and ${ }^{1+3} \mathrm{Xe}$ as deduced from the present work.

\section{Conclusion}

With a few exceptions, the level structure of the neutron-rich Xe isotopes do not display the characteristic pattern of reflection-asymmetric nuclei, but rather features related to an octupole softness. Therefore $Z=54$ may be considered as the lower boundary of the stable 


$$
-6-
$$

octupole deformation region with $\mathrm{Z} \approx 58, \mathrm{~N} \approx 88$ nuclei.

Eurogam is funded jointly by IN2P:3 (France) and the SERC (U.K.). This work was partially supported by the Science and Engineering Research Coucil of the U.K. under grant No. GR.H71161 and by the US Deparment of Energy under contracts No. W-31-109-ENG38 and No. DE-FG02-91ER-40609. The authors are also indebted for the use of ${ }^{248} \mathrm{Cm}$ to the Office of Basic Energy Sciences, US Department of Energy, through the transplutonium element production facilities at the Oak Ridge National Laboratory.

* Permanent address : Jagellonian University, Krakow, Poland.

$\ddagger$ Now at Argonne National Laboratory, Argonne, USA.

\section{References :}

1) W.R. Phillips et al., Phys. Rev. Let.t. 57 (1986) 3257.

2) W.R. Phillips et al., Phys. Lett. B212 (1988) 402.

3) S. Ćwiok and W. Nazarewicz, Nucl. Phys. A496 (1989) 367.

4) W. Nazarewicz and S.L. Tabor, Phys. Rev. C45 (1992) 2226.

$\left.{ }^{5}\right)$ V. Martín and L.M. Robledo, Phys. Rev. C48 (1993) 188.

$\left.{ }^{6}\right)$ A.S. Mowbray et al., Phys. Rev. C42 (1990) 1126.

7) C.W. Beausang et al., Nucl. Instr. Meth. A313 (1992) 37; F.A. Beck, Prog. Part. Nucl. Phys. 28 (1992) 443.

8) M.A.J. Mariscotti, Phys. Rev. Lett. 24 (1970) 1242.

$\left.{ }^{9}\right)$ G.A. Leander et al., Phys. Lett. B152 (1985) 284.

10) R.K. Sheline, Int. J. Mod. Phys. E2 (1993) 657.

\section{DISCLAIMER}

This report was prepared as an account of work sponsored by an agency of the United States Government. Neither the United States Government nor any agency thereof, nor any of their employees, makes any warranty, express or implied, or assumes any legal liability or responsibility for the accuracy, completeness, or usefulness of any information, apparatus, product, or process disclosed, or represents that its use would not infringe privately owned rights. Reference herein to any specific commercial product, process, or service by trade name, 'rademark, ence herein to any specific commerise does not necessarily constitute or imply its endorsement, recommendation, or favoring by the United States Government or any agency thereof. The views and opinions of authors expressed herein do not necessarily state or reflect those of the United States Government or any agelicy shereof. 

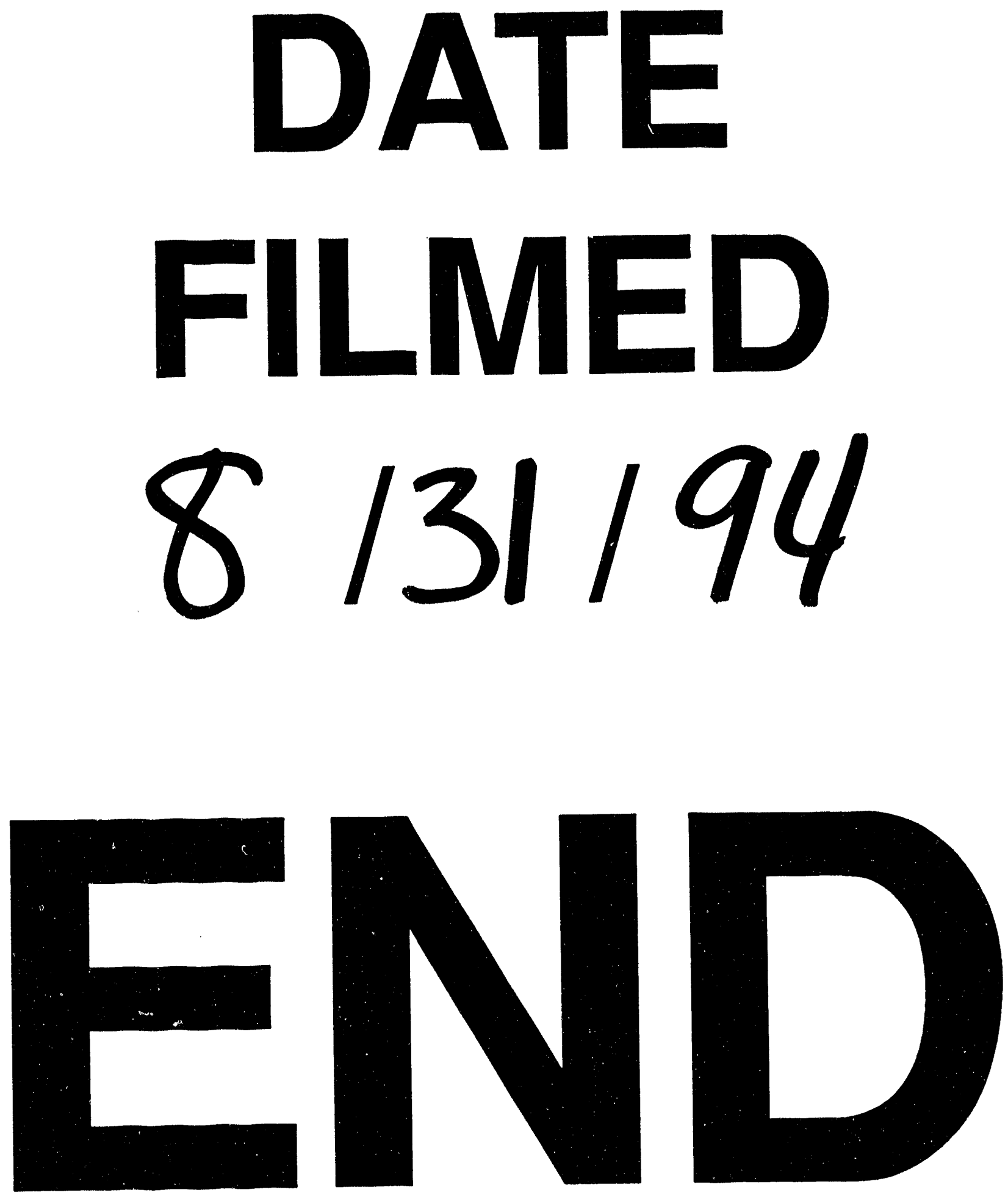


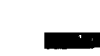

$$
\text { m }
$$$$
\text { . }
$$

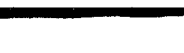

列

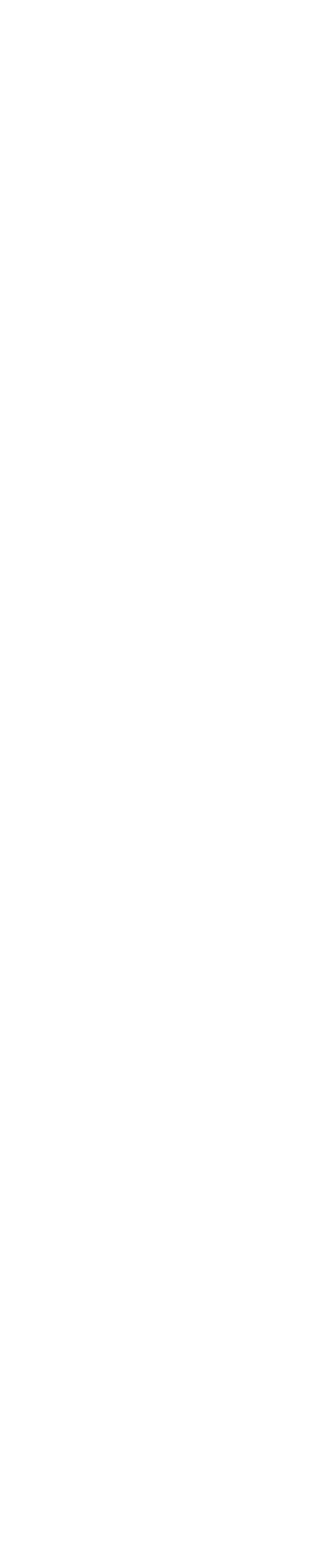

